\title{
Phenotypic and Cell Wall Proteomic Characterization of a DDR48 Mutant Candida albicans Strain
}

\author{
Pamela El Khoury ${ }^{1}$, Carell Salameh ${ }^{1}$, Samer Younes ${ }^{2}$, Andy Awad ${ }^{1}$, Yana Said ${ }^{1}$, and Roy A. Khalaf ${ }^{1 *}$ \\ ${ }^{1}$ Department of Natural Sciences, Lebanese American University, PO Box 36, Byblos, Lebanon \\ ${ }^{2}$ Department of Chemistry, Technical University of Munich, Lichtenbergstrabe 4, 85748, Munich, Germany
}

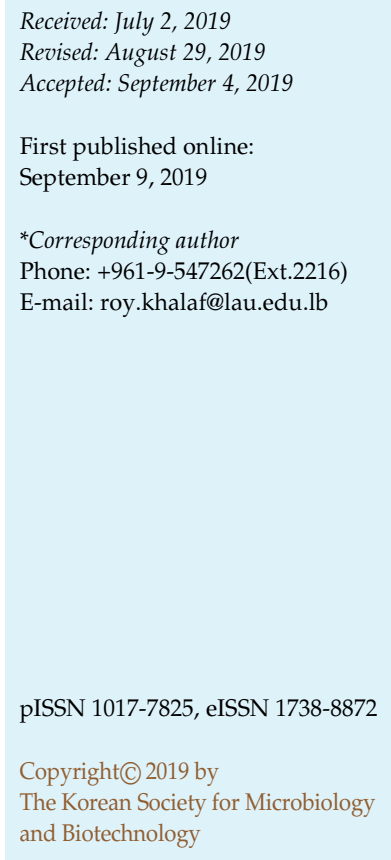

Candida albicans is an opportunistic fungus possessing multiple virulence factors controlling pathogenicity. Cell wall proteins are the most important among these factors, being the first elements contacting the host. Ddr48 is a cell wall protein consisting of 212 amino acids. A DDR48 haploinsufficient mutant strain was previously found necessary for proper oxidative stress response and drug resistance. In this study, we aimed to further elucidate the role of Ddr48 by performing additional phenotypic characterization assays. A combinatory proteomic and bioinformatics approach was also undertaken to determine differentially expressed cell wall proteins. Results showed that the mutant strain exhibited a $10 \%$ decrease in adhesion mirrored by a $20 \%$ decrease in biofilm formation, and slight sensitivity to menadione, diamide, and SDS. Both strains showed similar hyphae formation, virulence, temperature tolerance, and calcofluor white and Congo red sensitivities. Furthermore, a total of 8 and 10 proteins were identified exclusively in the wild-type strain grown under filamentous and nonfilamentous conditions respectively. Results included proteins responsible for superoxide stress resistance (Sod4 and Sod6), adhesion (Als3, Hyr4, Pmt1, and Utr2), biofilm formation (Hsp90, Ece1, Rim9, Ipp1, and Pra1) and cell wall integrity (Utr2 and Pga4). The lack of detection of these proteins in the mutant strain correlates with the observed phenotypes.

Keywords: Candida albicans, cell wall, biofilms, oxidative stress, tandem mass spectrometry

\section{Introduction}

Candida albicans is an opportunistic diploid fungal pathogen well known as a harmless colonizer of gut flora in healthy individuals that can turn pathogenic when the host's immunity is disturbed [1]. The virulent nature of this fungus is normally kept under surveillance by the host's immune system [2]. However, this benign colonization becomes pathogenic when immunity is compromised or when there is epithelial damage [1]. This is mainly caused by diabetes, chemo or radiotherapies, pregnancy, malignancy and neutropenia in addition to several other nosocomial factors such as hospital catheterization [3].

Infections caused by C. albicans are termed 'candidiasis' and can range from mild superficial infections to lifethreatening systemic infections [4]. Correct identification of C. albicans is crucial to reduce the emergence of novel resistant strains that can result from prescribing the wrong medications [5, 6]. Even with the use of aggressive therapeutic techniques and all the advancements in medical treatments, C. albicans infections have a poor prognosis with a mortality rate approaching fifty percent $[3,7]$. The exact pathway utilized by $C$. albicans to transform itself from the commensal state to the pathogenic state has been intensively studied [8]. C. albicans possesses multiple virulence factors expressed at different time-points where its ability to coordinate the expression of such factors in response to host defenses and changing environmental conditions is crucial in making it the successful pathogen that it is [9].

C. albicans is a dimorphic fungus since it is commonly isolated as either a round, yeast-shaped blastospore or a hyphal cell where the reversible transition between these two forms is termed 'dimorphic switching' [10]. Either form on its own is not sufficient for virulence; it is the 
switching between these two forms that establishes and maintains the virulence of $C$. albicans [11]. Dimorphic switching and hyphal growth are induced by various environmental factors that mimic physiological conditions of the host such as a temperature of $37^{\circ} \mathrm{C}$, nitrogen depletion, carbon dioxide availability, hypoxia, neutral to basic $\mathrm{pH}$, amino acid and peptidoglycan availability, contact with macrophages, and the presence of serum, the most potent hyphal inducer [12].

C. albicans cells are enclosed within a versatile cell wall that is highly adaptable to stresses and alterations in environmental conditions [13]. The cell wall is essential for maintaining structural integrity. It is responsible for adhesion, the initial step in causing an infection, and for anchorage of cell surface proteins that are necessary for virulence. It allows for dimorphism, stress tolerance, antigenicity and other virulence processes [14]. The cell wall is organized in a bi-layer structure: an inner stresstolerant chitin layer bound mainly to the $\beta-1,3$-glucan and some $\beta$-1,6-glucan molecules, and the outermost layer consisting of the mannoproteins [15]. Cell wall proteins (CWPs) are divided into two major groups based on their linkage and attachment site. GPI-anchored proteins are linked to the $\beta-1,6$-glucan layer through a glycosylphosphatidylinositol (GPI) anchor added post translationally to the C-terminus [16, 17]. Pir proteins (Proteins with Internal Repeats) are linked directly to the $\beta-1,3$-glucan layer via alkali-sensitive covalent bonding [16]. Most CWPs are considered virulence factors since they have roles in various pathogenicity mechanisms. Some are antigenic molecules that induce human immune responses. Some CWPs act as superoxide dismutases, heat shock proteins, and catalases that allow C. albicans to evade those responses by shielding it from the reactive oxygen species (ROS) burst $[6,18]$. Many CWPs have crucial roles in cell wall synthesis, growth, and overall virulence as deacetylases, hydrolases, hydrophobins, transglycosidases, and yapsins [13]. Many GPI-anchored proteins are adhesins [17]. Metal acquisition and $\mathrm{pH}$-sensing proteins are also incorporated within the cell wall along with efflux pumps that help the fungus get rid of the antifungal drugs and gain resistance [9]. As such, the cell wall and its proteins are considered potential drug targets given the prevailing resistance to the current limited number of antifungal drugs [19]. Therefore, achieving proper characterization of these CWPs is a key step in battling infections and creating innovative pharmacological drugs and antifungal therapies that target these proteins resulting in a fungus with attenuated virulence that cannot escape the immune system.
Ddr48, a stress-associated protein on the cell wall, is composed of 212 amino acids and is among the major immunodominant antigens expressed during candidiasis [20]. Ddr48 had been previously studied in our lab by generating a haploinsufficient DDR48 mutant strain. The strain was found to be slightly more susceptible to itraconazole, fluconazole, and ketoconazole than the wildtype strain. When tested for hydrogen peroxide tolerance, the mutant strain also showed higher sensitivity than the wild-type strain [21]. In a later study this protein was found to be necessary for DNA damage and oxidative stress induced by 4-nitroquinoline 1-oxide, flocculation, but not filamentation [22].

In this study, we aim to further elucidate the role of Ddr48 by subjecting our mutant strain to additional assays. Since Ddr48 is a cell wall protein we hypothesize that a mutant strain would have an impact on the proteomic constitution, architecture, and rigidity of the cell wall. Characterization was performed through classical phenotypic assays, and through a proteomic/bioinformatics approach; a method previously utilized by our lab to characterize DSE1, PGA1, PIR32, and HWP2 mutant C. albicans strains [23-26]. For phenotypic characterization, both wild type and mutant strains were tested for their tolerance to various stresses, and for their ability to form biofilms and adhere to surfaces. For proteomic characterization, CWPs from both strains were isolated and fractionated. Tandem mass spectrometry was later applied and resultant proteome profiles of the strains were compared to determine differentially expressed proteins that explain the observed phenotypic variations.

\section{Materials and Methods}

\section{Strains}

The C. albicans parental strain BWP17 + pABSK2 (ura3: : 1imm434) ura3: : 1imm434 his1: : hisG/ his1: : hisG arg4: : hisG/arg4: : hisG), and the mutant DDR48/ddr48::URA3+pABSK2 were utilized in this study [21]. The revertant strain (DDR48/ddr48: : HIS1+ pABSK2DDR48) was also utilized [21].

\section{Media Preparation and Culture Conditions}

Rich potato dextrose agar (PDA) medium (HiMedia, India) was used to grow strains during routine culturing and for oxidative stress tolerance, cell surface disruption resistance, temperature sensitivity, and adhesion experiments. PDA plates were supplemented with histidine and uracil throughout all phenotypic characterization experiments. For the biofilm assay, yeast nitrogen base (YNB) synthetic medium (Fluka, Switzerland) was supplemented with uracil and histidine. For routine growth to exponential phase 
for oxidative stress tolerance, cell surface disruption resistance, temperature sensitivity, and adhesion experiments, rich potato dextrose broth (PDB) liquid media (Hi Media, India) was used, and strains were incubated at $30^{\circ} \mathrm{C}$ under aerobic conditions.

For proteomic analysis, strains were grown in PDB media supplemented with histidine and uracil until exponential phase. For filamentous growth, PDB was supplemented with $10 \%$ fetal bovine serum (FBS) and incubated at $37^{\circ} \mathrm{C}$ under aerobic conditions.

\section{Phenotypic Characterization}

Oxidative stress tolerance. During exponential growth phase, 4 -fold serial dilutions $\left(10^{5}\right.$ to $10^{2}$ cells $\left./ \mathrm{ml}\right)$ were prepared and treated with hydrogen peroxide $(10,25$, or $50 \mathrm{mM})$ for one hour followed by spotting $(5 \mu \mathrm{l})$ onto PDA plates. Untreated cultures were also spotted as controls. Growth was monitored for three days [27]. The same was applied to test the tolerance of the strains to menadione $(0.5,1$, and $1.5 \mathrm{mM})$ and diamide $(2,4$, and $10 \mathrm{mM})$.

Heat shock tolerance. A 4-fold serial dilution was performed for each strain. Strains were grown at $28^{\circ} \mathrm{C}$ and then heat shocked for three hours at $42^{\circ} \mathrm{C}$ before incubation at $28^{\circ} \mathrm{C}$ for $20 \mathrm{~min}$. Five microliters of each strain was spotted on PDA plates and growth was monitored for three days [28].

Osmotic stress tolerance. A 4-fold serial dilution was performed for each of the tested strains during their exponential growth phase. The strains were then treated with different concentrations of $\mathrm{NaCl}(10,20$, or $30 \mathrm{mg} / \mathrm{ml})$ to test the ability of the strains to resist osmotic stress. Strains were incubated at $37^{\circ} \mathrm{C}$ for $2.5 \mathrm{~h}$, and $5 \mu \mathrm{l}$ of each was subsequently spotted on PDA plates. Growth was monitored for three days [28].

Resistance to cell surface disrupting agents. The strains were grown until exponential phase and subjected to a 4 -fold serial dilution. Five microliters of the serial dilutions was spotted on PDA plates treated with either $0.05 \%, 0.1 \%$, and $0.2 \%$ SDS (SigmaAldrich, Germany), 100 to $300 \mu \mathrm{g} / \mathrm{ml}$ calcofluor white (SigmaAldrich), or 100 to $300 \mu \mathrm{g} / \mathrm{ml}$ Congo red (Sigma-Aldrich). The plates were incubated at $30^{\circ} \mathrm{C}$ for 3-4 days [29].

Biofilm formation. The ability of the mutant and wild-type strains to form biofilms on polystyrene microtiter wells was assayed according to a previously described protocol [30]. Briefly, $5 \times 10^{6}$ cells of each of the strains were incubated in a flatbottomed microtiter well pretreated with $5 \%$ serum overnight. Strains, along with an un-inoculated control well, were left to incubate at $37^{\circ} \mathrm{C}$ in a shaking incubator at $75 \mathrm{rpm}$ for two hours. Wells were rinsed with $\mathrm{PBS}$ to remove non-adherent cells followed by the addition of YNB and incubation at $37^{\circ} \mathrm{C}$ for $48 \mathrm{~h}$. After incubation, plates were washed again, fixed with methanol, and air dried. A $0.2 \%$ crystal violet solution was added and left for $20 \mathrm{~min}$. Excess crystal violet was washed with distilled water and the crystal violet bound to biofilm cells was released by acetic acid treatment. Absorbance of the released crystal violet was measured using a spectrophotometer at $590 \mathrm{~nm}$ wavelength.

Adhesion assay. Adhesion ability of the mutant and wild-type strains was assayed against human epithelial cell line HT-29 as previously described [31]. Briefly, around 100 cells of each of the wild-type and mutant strains were incubated with human cell lines in 6-well microtiter plates for 180 minutes followed by washing with PBS and overlaid with molten PDA agar. Colonies were counted after overnight growth and then compared with a control plate inoculated with the same number of $C$. albicans cells. The results of this experiment are expressed as percentage adhesion of the control plate [32].

Chitin content assay. Cell wall chitin content of the mutant and wild-type strains was measured according to a modified protocol described previously [33]. Briefly, $6 \mathrm{~N} \mathrm{HCl}$ was used to hydrolyze $50 \mathrm{mg}$ of wet weight purified cell walls at $100^{\circ} \mathrm{C}$ overnight. After centrifugation, the pellet was reconstituted in $1 \mathrm{ml}$ of distilled water. A $0.1 \mathrm{ml}$ aliquot of this sample was added to $0.1 \mathrm{ml}$ of solution $\mathrm{A}\left(1.5 \mathrm{~N} \mathrm{Na}_{2} \mathrm{CO}_{3}\right.$ in $4 \%$ acetylacetone). The mixture was incubated at $100^{\circ} \mathrm{C}$ for $20 \mathrm{~min}$, and after cooling to room temperature, $0.7 \mathrm{ml}$ of $96 \%$ ethanol was added to the mixture followed by addition of a $0.1 \mathrm{ml}$ of solution B (1.6 g of $p$-dimethylaminobenzaldehyde in $30 \mathrm{ml}$ of concentrated $\mathrm{HCl}$ and $30 \mathrm{ml}$ of $96 \%$ ethanol). The mixture was incubated for one hour at room temperature, and absorbance of the samples was measured spectrophotometrically at $520 \mathrm{~nm}$. The results were plotted against a standard curve generated by the use of known glucosamine standards taken through the same procedure as our samples. Chitin amount was expressed as a percentage of the wild-type strain [28].

Virulence test: murine model of disseminated candidiasis. Six female BALB/c mice (Lebanese American University stock), four to six weeks old and weighing $20 \mathrm{~g}$ to $30 \mathrm{~g}$, were injected via the lateral tail vein with $200 \mu \mathrm{l}$ of $1.5 \times 10^{8}$ cells of the mutant strain suspended in PBS solution, pH 7.5. The same was applied for another six mice injected with cells of the wild-type strain instead. All 12 mice were given food and water ad libitum and monitored three times daily for survival over a period of 15 days [32]. Ethical guidelines as set by the NIH's Guide for the Care and Use of Laboratory Animals were followed and moribund mice were euthanized by $\mathrm{CO}_{2}$ inhalation.

Statistical analysis. All assays were performed in triplicates. The Student's t-test was used for the adhesion, biofilm, and chitin content assays. For the virulence assay, survival analysis was based on the Kaplan-Meier method and the log Rank test. A $p$ value of less than 0.05 was considered significant.

\section{Proteomic Characterization}

Cell wall isolation and protein extraction. Eight independent cell wall extractions from each strain and for each condition (filamentous and non-filamentous) were performed as described previously [24]. A minimum weight of $50 \mathrm{mg}$ of cultured cells was collected and the cells were first washed with water, spun at $4,000 \times g$ for five minutes, re-suspended in $5 \mathrm{ml}$ Tris $(5 \mathrm{mM}$, $\mathrm{pH}=7.8$ ), and divided into $1 \mathrm{~mL}$ Eppendorf tubes. They were spun again for three minutes at $12,000 \times g$. The supernatants were 
discarded and the pellets were resuspended in $750 \mu \mathrm{l}$ Tris $(5 \mathrm{mM}$, $\mathrm{pH}=7.8$ ). Then, $6 \mu$ l Protease Inhibitor Cocktail (Abcam ab65621) and cold glass beads were added and 30 vortexing cycles were performed for breakage as follows: $30 \mathrm{sec}$ on vortex then $30 \mathrm{sec}$ on ice. Breakage was indicated by the orange color of the sample due to a reaction of acidic cytosol with the Protease inhibitor, and its efficiency was monitored under the microscope. The solution on top of the beads was poured into new pre-weighed tubes and the beads were washed several times with cold $\mathrm{NaCl}(1 \mathrm{mM})$ to collect as much cell wall material and proteins as possible. The samples were spun at 3,000 $\times g$ for five minutes and the supernatant of each tube now containing intracellular proteins was poured off while the pellet was re-suspended in $\mathrm{NaCl}(40 \mathrm{ml}, 1 \mathrm{mM})$ and spun. The wash step was repeated at least three times. SDS extraction buffer (50 mM Tris, 2\% SDS, $100 \mathrm{mM} \mathrm{Na-EDTA,} 150 \mathrm{mM} \mathrm{NaCl}, \mathrm{pH} 7.8$ ) with $\beta$-ME ( $8 \mu \mathrm{l}$ per $1 \mathrm{ml}$ SDS extraction buffer) was added $(0.5 \mathrm{ml}$ buffer per $100 \mathrm{mg}$ wet weight walls) and the tubes were boiled for $10 \mathrm{~min}$ followed by centrifugation for five minutes at 3,000 $\mathrm{xg}$. The supernatant was collected and analyzed for SDS extractable proteins. SDS extraction buffer and $\beta$-ME were added as before to re-suspend the pellet. Samples were boiled then cooled to room temperature for three more cycles, and finally spun for five minutes at 3,000 $\times g$ where the resulting pellet was suspended in water. Wash steps with water (Type 2) were performed to remove excess SDS. The final pellet was frozen in liquid nitrogen and freeze-dried. Lyophilized cell walls were stored at $-20^{\circ} \mathrm{C}$ until use.

Extraction of alkali labile CWPs. The cell wall pellets were subjected to overnight incubation with $\mathrm{NaOH}(30 \mathrm{mM})$ at $4^{\circ} \mathrm{C}$. They were then neutralized with aqueous acetic acid $(30 \mathrm{mM})$. Samples were spun, and supernatants were collected and subjected to tryptic digestion.

Glucanase treatment of cell wall pellets. Cell wall pellets were incubated overnight at $37^{\circ} \mathrm{C}$ with $1 \mathrm{mg}$ of glucanase in sodium acetate buffer $(1 \mathrm{ml}, 150 \mathrm{mM}, \mathrm{pH}=5)$ per $10^{8}$ cell equivalents [34]. Spectrophotometric analysis at $600 \mathrm{~nm}$ was used to estimate cell numbers. Supernatants were collected and subjected to tryptic digestion.

Tryptic digestion. The cell wall extracts were incubated in a reducing buffer $\left(10 \mathrm{mM} \mathrm{DTT}, 100 \mathrm{mM} \mathrm{NH}_{4} \mathrm{HCO}_{3}\right)$ at $55^{\circ} \mathrm{C}$ for one hour. Samples were cooled to room temperature and spun. An alkylating buffer ( $65 \mathrm{mM}$ iodoacetamide, $100 \mathrm{mM} \mathrm{NH}_{4} \mathrm{HCO}_{3}$ ) was added to the pellets that were kept for $45 \mathrm{~min}$ at room temperature in the dark. Subsequently, a quenching solution (55 mM DTT, 100 $\mathrm{mM} \mathrm{NH} \mathrm{NH}_{4} \mathrm{HCO}_{3}$ ) was added to the samples for five minutes at room temperature. Ammonium bicarbonate buffer $(50 \mathrm{mM})$ was used to wash the samples five times. Pellets were re-suspended in solution containing ammonium bicarbonate $(50 \mathrm{mM})$ and trypsin $(1 \mu \mathrm{g} / \mu \mathrm{l})$. Samples were left at $37^{\circ} \mathrm{C}$ for $16 \mathrm{~h}$. Then, they were spun, and the supernatants were collected and prepared for Zip Tipping by adding TFA $(0.1 \% \mathrm{~V} / \mathrm{V})$.

Peptide concentration. ZipTip C18 clean up tips were wetted in acetonitrile solution and then equilibrated in a $0.1 \%$ TFA HPLC water solution. Sample binding was achieved by full pressing the pipette a minimum of 10 times in the digest tube. The membrane was then washed in a $0.1 \%$ TFA HPLC water solution. Sample elution was performed using $10 \mu \mathrm{l}$ of elution buffer $(0.1 \%$ TFA (v/v) in HPLC water/acetonitrile (1:1)).

Mass spectrometry. Digested proteins were spotted on a stainless steel target plate (Opti-TOF TM 384 Well Insert, 128x81 mm RevA, Applied Biosystems) and overlaid with $\alpha$-cyano-4hydroxy-cinnamic acid matrix solution (10 mg CHCA matrix in $50 \%$ acetonitrile with $0.1 \%$ TFA) and air-dried. MALDI-TOF-TOF MS spectra were acquired using the 4800 MALDI-TOF-TOF analyzer (operated by the 4000 Series Explorer software version 3.7). The instrument was externally calibrated using TOF/TOF Calibration Mixture (Mass Standards Kit for Calibration of $A B$

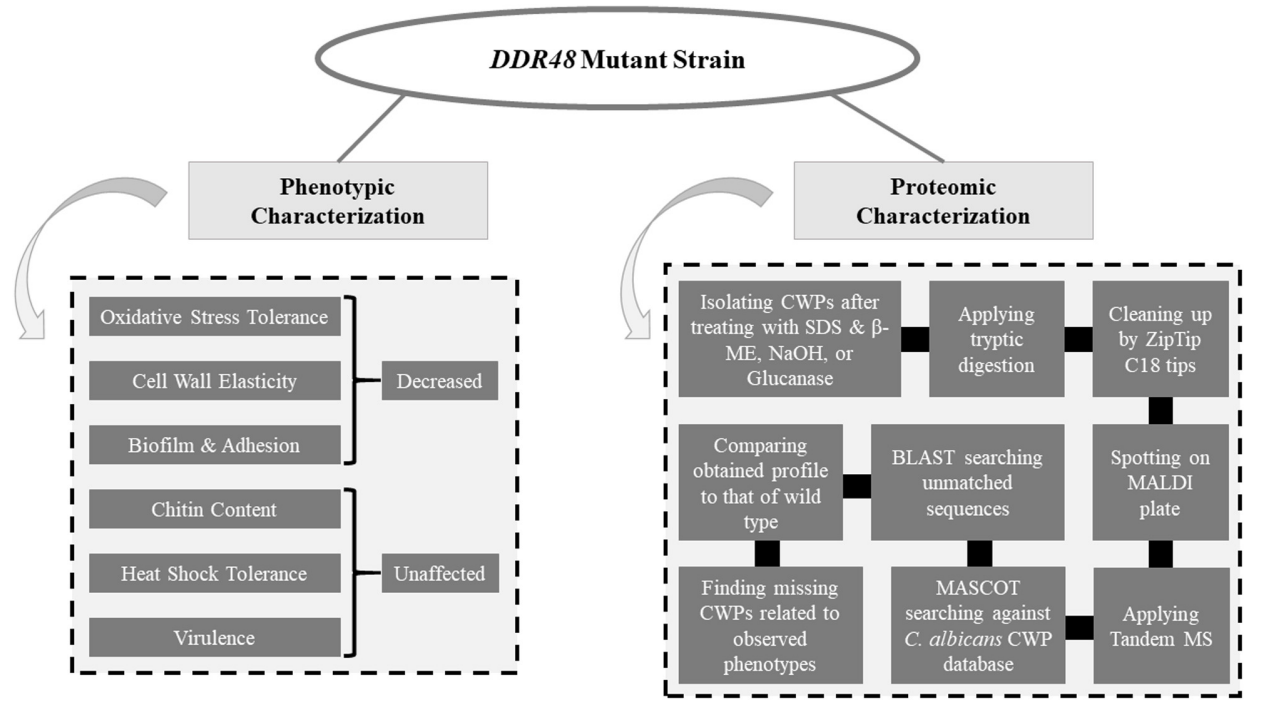

Fig. 1. Methodology flowchart.

A combinatory phenotypic and proteomic approach was utilized to achieve characterization of Ddr48. 
SCIEX TOF/TOF Instruments). The chosen mass range was $499 \mathrm{Da}-2,500 \mathrm{Da}$ and the focus mass was 1,500 Da. The resulting mass lists were manually scanned for known contaminant mass peaks (keratin, matrix, and trypsin autolysis) to create an exclusion list that was applied in the acquisition method used for the all MS/MS data acquisitions performed. In addition, the common Repository of Adventitious Proteins, cRAP, was utilized to remove common contaminants.

Protein identification. MS/MS Ion Search was performed first using the contaminants and cRAP databases, in order to eliminate any additional contaminants whose peaks were not included within the exclusion list, then we used a custom C. albicans CWP database on the MASCOT Server in order to identify the proteins within the samples as described previously [25]. The database consisted of protein sequences of all curated C. albicans proteins available in the Swissprot database with gene ontology related to cell wall, plasma membrane, and transmembrane localization tags. The peptide and fragment tolerance values in the MASCOT Server's search parameters page were specified at $\pm 2 \mathrm{Da}$ each. Carbamidomethyl $\mathrm{C}$ was chosen as a fixed modification, whereas Oxidation at $\mathrm{M}$ was selected as a variable modification. Up to two missed cleavages were permitted for trypsin. A peptide charge of 1+ was assigned and MALDI-TOF-TOF was picked in the instrument type option.
It is important to note that only proteins appearing in half of the extractions or having a minimum of $2 \%$ sequence coverage (equating to an average of 12 amino acids identified in the exact order) were considered successful hits [35]. All peptide sequences identified by MASCOT but unassigned to any protein were BLAST searched on Candidagenome.org where the selected target genome and target sequence dataset were "Candida albicans SC5314 Assembly 22" and "Proteins - translation of coding sequence (PROTEIN)" respectively and no gapped alignments were allowed. The cutoff e-value was set at $<0.05$ for both MASCOT and BLAST searches. Fig. 1 summarizes the assays and procedures utilized in this study.

\section{Results}

\section{Phenotypic Characterization}

The wild-type and mutant strains were subjected to a battery of tests at different concentrations. For each test a representative picture is shown in Fig. 2.

Oxidative stress tolerance. The C. albicans wild-type and mutant strains showed varying tolerance capacities to the different oxidative stress agents. The mutant strain was found to be slightly, yet consistently, more sensitive to

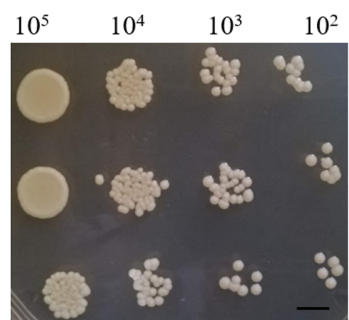

A
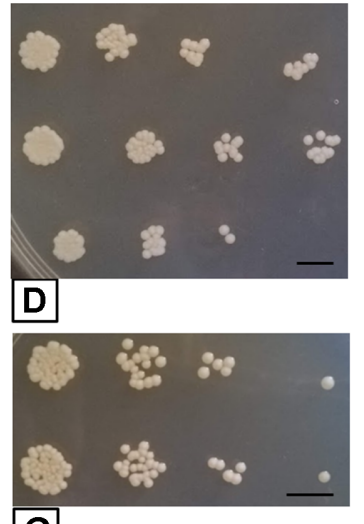

G

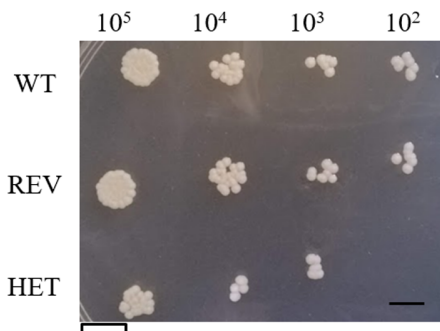

B
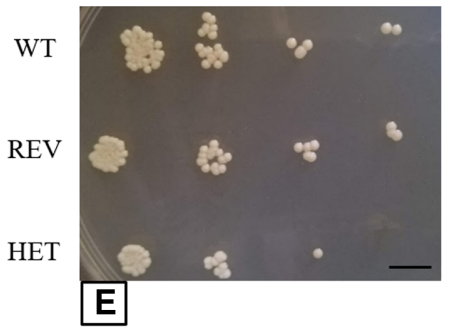

WT
HET

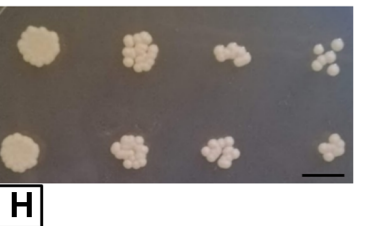

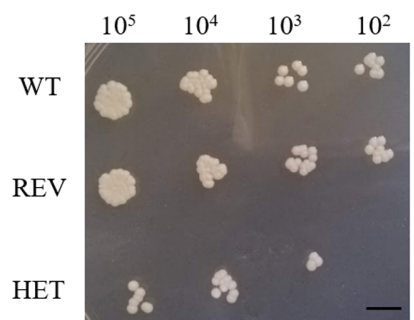

C
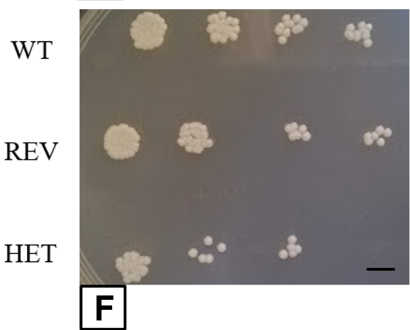

WT

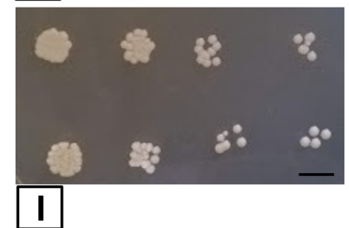

Fig. 2. Resistance to stress.

(A) Control, untreated cells. (B) $10 \mathrm{mM}$ hydrogen peroxide. (C) $0.5 \mathrm{mM}$ menadione. (D) $2 \mathrm{mM}$ diamide. (E) $0.05 \% \mathrm{SDS}$. (F) $10 \mathrm{mg} / \mathrm{ml} \mathrm{NaCl}$. (G) Heat shock at $42^{\circ} \mathrm{C}$. (H) $100 \mu \mathrm{g} / \mathrm{ml}$ Congo red. (I) $100 \mu \mathrm{g} / \mathrm{ml}$ calcofluor white. Note that the mutant strain is more sensitive to oxidative stress agents, osmotic stress, and SDS than the wild type whereas the revertant strain restored growth under these stresses, and that the mutant and wild-type strains can equally tolerate heat shock, Congo red, and calcofluor white. 


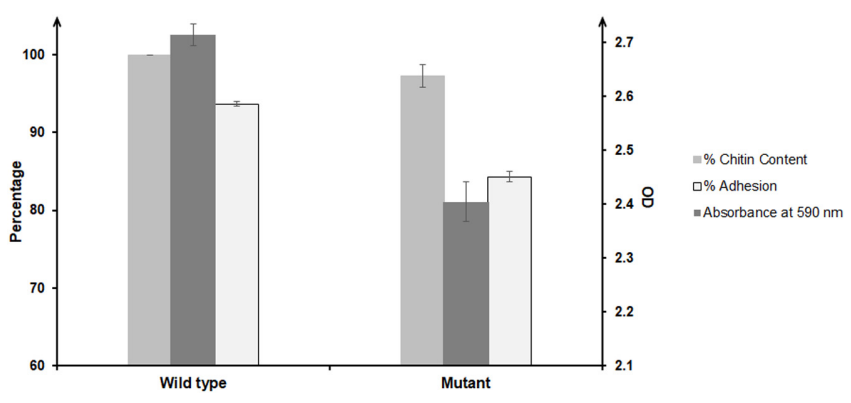

Fig. 3. Pathogenicity related attributes.

No significant difference between the mutant and wild type strains was shown as far as ability to form cell wall chitin (left column, $p$-value $=0.1404$ ). Our mutant however was slightly defective in adhesion with a significant $10 \%$ reduction in adhesion to human epithelial cells (right column, $p$-value $=0.0002$ ). . The decrease in adhesion was mirrored by a significant $15 \%$ decrease in biofilm production seen as a decrease in crystal violet absorbance at $590 \mathrm{~nm}$ (middle column, $p$-value $=$ 0.03 ). Mutant values for chitin content are expressed as percentage of the wild-type strain. All experiments were performed in triplicates with error bars representing the average along with the -/+ SEM.

hydrogen peroxide, menadione, and diamide than the wild-type strain on all concentrations tested while the revertant strain restored normal cell growth as seen in Figs. 2B, 2C, and 2D.

Heat shock tolerance. Both strains were also tested for their ability to endure an increase in temperature by subjecting cells to a heat shock of $42^{\circ} \mathrm{C}$ for three hours. Results in Fig. 2G show no significant difference between the mutant and wild-type strains, indicating equal resistance to heat shock.

Osmotic stress tolerance. Upon treatment with different concentrations of $\mathrm{NaCl}$ to investigate tolerance to osmotic stress, the mutant strain was slightly more sensitive to osmotic pressure created by the salt gradient than the wild type. The revertant strain showed restored resistance to osmotic stress as seen in Fig. 2F.

Resistance to cell surface disrupting agents. In order to assess the role of Ddr48 in resisting cell surface disrupting agents, strains were treated with varying concentrations of SDS, calcofluor white and Congo red. The mutant strain showed higher sensitivity to SDS when compared to the wild-type and revertant strains as seen in Fig. 2E. However, no significant difference was observed between the mutant and wild-type strains when treated with calcofluor white or Congo red as shown in Fig. 2H and Fig. 2I.

Biofilm formation. The ability of the strains to form biofilms on polystyrene microtiter wells was assayed and the mutant strain exhibited a significant $20 \%$ decrease in

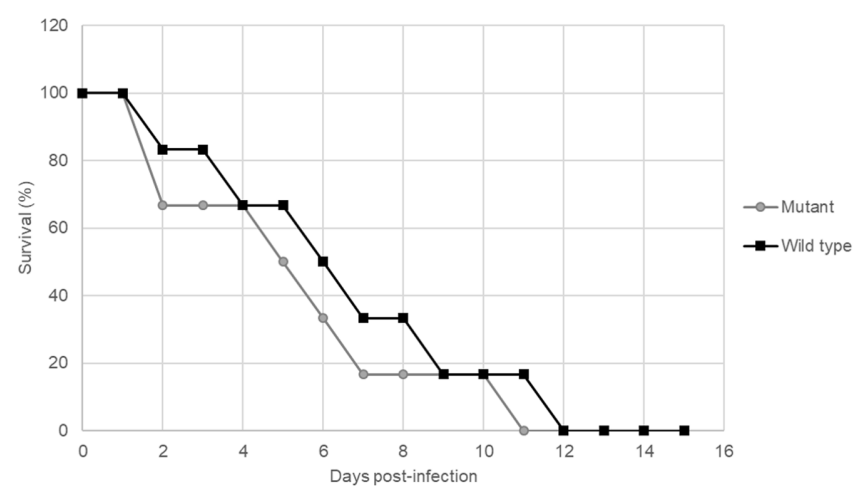

Fig. 4. Virulence test.

For each strain, six mice were intravenously injected in the tail with Candida albicans and survival was monitored for 15 days. Both strains were able to cause disseminated candidiasis and kill mice within 2 weeks of infection, however, the mutant exhibited a slight-but-notstatistically-significant delay in causing death $(p$-value $=0.4)$.

biofilm forming capacity as shown in Fig. 3 reflecting a decrease in absorbance of crystal violet.

Adhesion assay. Adhesion ability of both strains was assayed against human epithelial cell line HT-29. The mutant strain was significantly $10 \%$ less adhesive than the wild type as seen in Fig. 3.

Chitin content assay. The amount of cell wall chitin was determined spectrophotometrically following acid hydrolysis. No significant variation was observed between the mutant and the wild type as seen in Fig. 3.

Virulence test. Virulence capabilities of both strains were tested via a murine model of disseminated candidiasis. As shown in Fig. 4, infection with the mutant strain led to a slight delay in mice death however the delay was not significant as all mice died within the same two weeks post infection period as the wild type.

\section{Proteomic Characterization}

The cell wall proteome profiles of the mutant and the wild-type strains were compared in order to detect differentially expressed CWPs responsible for the previously described phenotypes. It is important to note that in our study, the main focus is on the proteins exclusive to the wild type and lacking in the mutant strain since the latter was found to be more attenuated in our phenotypic studies.

Proteins detected solely in the wild-type strain under filamentous growth conditions. Proteins that were exclusively detected in the wild type are listed in Table 1 and mainly function in adhesion (Hyr4 and Pra1) and biofilm formation (Rim9, Ece1, Ipp1, Pmt1, and Pra1). The 
Table 1. Proteins exclusive to the wild-type strain grown under filamentous conditions: Proteins were detected either via MASCOT as shown through sequence coverage, or via BLAST as shown through an E-value.

\begin{tabular}{llcclc}
\hline Protein & \multicolumn{1}{c}{ Protein description } & $\begin{array}{c}\text { Sequence } \\
\text { coverage }(\%)\end{array}$ & $\begin{array}{c}\text { Number of } \\
\text { missed cleavages }\end{array}$ & Peptide sequence & E-value \\
\hline Cdc42 & Rho-type GTPase & - & - & AVKYVECSALTQR & $5.00 \mathrm{E}-08$ \\
Ece1 & Candidalysin, cytolytic peptide toxin & - & - & RDGANDDVANAVVR & $3.00 \mathrm{E}-08$ \\
Hyr4 & Putative GPI-anchored adhesin-like protein & - & - & VTHFTNSSTSNR & $4.00 \mathrm{E}-07$ \\
Ipp1 & Inorganic pyrophosphatase & 3.8 & 0 & TILNMIVEVPR & - \\
Pmt1 & Protein mannosyltransferase & - & - & MAKKPVTPASKVAAK & $1.00 \mathrm{E}-09$ \\
Pra1 & pH-regulated antigen & 4 & 0 & TNIFWAGDLLHR & - \\
Rim9 & pH-response regulator protein palI/RIM9 & 2.9 & 1 & YANDDMRIMR & - \\
\hline
\end{tabular}

lack of these proteins from the mutant strain explains its reduced ability to adhere, and to form biofilms.

Proteins detected solely in the wild-type strain under non-filamentous growth conditions. Proteins functioning in adhesion (Als3 and Utr2), biofilm formation (Als3 and Hsp90), resisting oxidative stress (Sod4, Sod6, and Vma2), and cell wall rigidity (Utr2 and Pga4) were exclusively identified in the wild-type strain, which showed better abilities than the mutant strain. Table 2 lists all the proteins solely detected in the wild-type strain grown under nonfilamentous conditions. The absence of these proteins from the mutant strain reflects its impaired phenotypes.

\section{Discussion}

This current study aimed at further elucidating the role of Ddr48 in C. albicans by subjecting our strongly haploinsufficient $D D R 48$ heterozygote mutant strain to further tests. Dib et al. found the mutant strain to be more sensitive to oxidative stress; which was seen as a decrease in OD growth in liquid upon addition of hydrogen peroxide [21]. The Cleary group then demonstrated that the $d d r 48$ mutant strain was more sensitive to oxidative stress imposed by 4-nitroquinoline 1-oxide [22]. As such, we decided to perform additional oxidative stress tolerance tests by agar spotting. Our mutant strain turned out to be slightly more sensitive to hydrogen peroxide by such a method, confirming our previous results. To test if this response is specific to hydrogen peroxide or a global response to oxidative stress, we then tested for resistance to two additional oxidative stress agents: menadione and diamide. The strain was found to be slightly more sensitive

Table 2. Proteins exclusive to the wild-type strain grown under non-filamentous conditions: Proteins were detected either via MASCOT as shown through sequence coverage, or via BLAST as shown through an E-value.

\begin{tabular}{|c|c|c|c|c|c|}
\hline Protein & Protein description & $\begin{array}{c}\text { Sequence } \\
\text { coverage (\%) }\end{array}$ & $\begin{array}{c}\text { Number of } \\
\text { missed cleavages }\end{array}$ & Peptide sequence & E-value \\
\hline \multirow[t]{2}{*}{ Als3 } & Agglutinin-like protein & 2 & 0 & GYLTDSR & - \\
\hline & & & 1 & CFKAGTNTVTFNDGDK & - \\
\hline Dfg5 & N-linked mannoprotein of cell wall and membrane & - & - & NSISNGCLFHLAAR & 3.00E-09 \\
\hline Eft2 & Elongation Factor 2; GTPase & - & - & DSVVAAFQWATK & 4.00E-07 \\
\hline \multirow[t]{2}{*}{ Hsp90 } & Heat shock protein & 3.5 & 1 & ELISNASDALDKIR & - \\
\hline & & & 0 & FTVTLDETNER & - \\
\hline Pga4 & Beta-1,3-glucanosyltransferase & - & - & AGIYVILDVNTPHSSITR & $4.00 \mathrm{E}-13$ \\
\hline Sod4 & Cell surface super oxide dismutase & - & - & SNSTSSSSSSSK & $1.00 \mathrm{E}-05$ \\
\hline Sod6 & Cell surface superoxide dismutase [Cu-Zn] & 3.8 & 0 & DEGPFFYHIHER & - \\
\hline Utr2 & Putative GPI anchored cell wall glycosidase & - & - & MSTFQESFDSK & $5.00 \mathrm{E}-06$ \\
\hline Vma2 & V-type proton ATPase subunit B & 2.3 & 1 & ISPKILEELYGR & - \\
\hline
\end{tabular}


to these agents than the wild type. The revertant strain restored normal cell growth and resistance to these three oxidative stress agents. Hydrogen peroxide, menadione, and diamide are used in our study to mimic the harsh conditions C. albicans faces when subjected to ROS bursts by macrophages [11]. Our experiments confirm that Ddr48 is involved in oxidative stress tolerance.

The wild type and mutant were also subjected to stresses induced by cell surface perturbing agents. The rationale behind such an experiment is that deletion of cell wall proteins has been previously shown to affect cell surface architecture and rigidity as in many instances it generates a less rigid cell wall, or alternatively the cell overcompensates for the deletion by turning on salvage pathways and increasing the chitin content resulting in an increasingly rigid cell wall leading to increased resistance to cell surface disrupting agents [28]. We found that our mutant strain was slightly less resistant to SDS than the wild type and that the revertant strain showed restoration of SDS resistance. This implies that Ddr48 is involved in protection against cell wall stress. Both strains however were equally sensitive to calcofluor white and Congo red. These agents have distinct modes of action since SDS affects and solubilizes the plasma membrane proteins [29], whereas Congo red and calcofluor white act on $\beta$-glucans and chitin fiber assembly respectively [36,37]. A recent study showed that $C$. albicans' ability to withstand hyperosmotic stress is controlled by the extent of its cell wall elasticity and crosslinking as the deletion of crosslinking enzymes induced susceptibility to osmotic stress [38]. We thus hypothesize that Ddr48 does not interfere with the formation and assembly of glucans or chitin fibers, but it might affect the crosslinking of these cell wall components altogether, thereby affecting the overall cell wall elasticity and integrity as observed by decreased resistance to SDS. We then performed a chitin content assay and, as suggested by the calcofluor and Congo red spotting tests, the cell wall chitin content did not vary between the wild type and the mutant.

In our study, we also tested both strains for their tolerance to heat shock with no discernable differences observed between both strains implying that Ddr48 is not required for thermal resistance. The ability of both strains to form biofilm was also assessed. We found the mutant strain to be slightly deficient in biofilm formation. Knowing that adhesion is an essential step in biofilm formation [39], we also performed an adhesion assay on human epithelial cells. As expected, the mutant strain mirrored the biofilm data and showed a significant drop in adhesion as well. This is in line with the observation of Cleary et al. [22] that a $d d r 48$ deficient strain fails to self-aggregate and flocculate properly. We thus confirm that Ddr48 affects adhesion and biofilm formation in C. albicans, probably indirectly by affecting the cell wall architecture and cell wall proteome.

Moreover, virulence was assayed through a murine model of disseminated candidiasis. All infected mice died within two weeks post intravenous injection, with a slight yet statistically insignificant delay in the mutant strain. This shows that Ddr48 does not play a significant role in virulence. The slight drop in biofilm formation, adhesion, and tolerance to stresses mentioned above was not enough to impart an attenuated virulence trait to the mutant.

We then proceeded to analyze the cell surface proteome of the mutant and wild-type strains to determine possible changes in the cell surface proteome that can explain the observed phenotypic results. It is interesting to note that the number of differentially detected proteins observed was relatively low compared to other studies with similar techniques [23-25]. This makes sense in fact and can be explained by the fact that our mutant phenotypes were not severe. It is noteworthy in this proteomic approach that the lack of detection of a particular protein in a sample does not necessarily mean that the protein is absent or not expressed. The protein could still be present in the sample but at an insufficiently low concentration for it to be successfully detected. We performed eight independent cell wall extractions for each strain.

Three proteins detected exclusively in the wild-type strain have functions in combating oxidative stress: Sod4, Sod6, and Vma2. Sod4 and Sod6 are superoxide dismutases that function in converting the damaging superoxide radicals generated by the host's macrophages and neutrophils into less harmful hydrogen peroxide that can be later transformed into water by catalases [40]. Vma2, a subunit of the vacuolar ATPase complex involved in the acidification of intracellular organelles, is needed for the resistance of $C$. albicans to oxidative stress [41]. The lack of detection of these three proteins from the mutant can be correlated with the reduced ability of this strain in surviving oxidative stress.

The extent of cell wall elasticity and integrity reflects the ability of $C$. albicans to tolerate cell wall perturbing agents and osmotic stress [38]. Pga4 is needed for the elongation of the $\beta-1,3$-glucan chains during cell wall biosynthesis and morphogenesis [29]. Utr2 is an extracellular glycosidase that links $\beta$-glucan to chitin thus affecting osmotic stress 
resistance [38, 42]. Dfg5 is a GPI-linked cell surface protein that functions in cell wall biogenesis and integrity [43]. These proteins were only detected in the wild type which has a more rigid cell wall and higher tolerance to osmotic and some cell wall perturbing agents than the mutant strain.

Biofilm formation is a multi-step mechanism in which various proteins and factors are recruited. Als3 is a cell surface adhesin that has a major role in yeast aggregation and consequently in biofilm formation [44]. Hsp90 is a heat shock protein that is a prime regulator of antifungal tolerance and dispersion of C. albicans biofilms [45]. Ece1 is a secreted protein contributing to biofilm formation [46]. Ipp1, an inorganic pyrophosphatase, and Pra1 are components of the biofilm matrix with Pra1 being a major constituent $[45,47]$. Pmt1 is a mannosyltransferase required for the early stages of biofilm formation [47]. Rim9 is a pHsensing protein that is biofilm induced $(45,49]$. Cdc42 is a Rho-type GTPase functioning in morphogenesis induced by a biotic stimulus and in maintaining hyphae; essential processes in biofilm formation [39, 50]. None of these proteins were detected in the mutant, which explains its reduced ability to form biofilms.

In C. albicans, Als3 is the chief adhesin and invasin [51]. Hyr4 is a GPI-anchored protein that aids the fungus in adhering to host tissues [17]. Pmt1 also functions in the adhesion of $C$. albicans to epithelial cells [48]. These adhesion proteins were not detected in any protein sample isolated from the mutant strain which showed reduced adhesion ability.

In addition to the above-mentioned proteins, Eft 2 and Tim21 were solely detected in the wild-type strain. However, these proteins are ribosomal and mitochondrial proteins respectively as referenced in candidagenome.org and uniprot.org. These are artifacts or contaminants from the cytosolic proteins during cell wall isolation. Additional contaminants such as these were detected exclusively in the mutant strain: Mic60, Pam17, Alo1, and Erv25.

In this study, we further characterized the C. albicans cell wall protein Ddr48. Our current study found the DDR48 mutant strain to have reduced resistance to oxidative stress, weakened cell wall elasticity as revealed by its increased sensitivity to SDS and osmotic stress, and decreased adhesion and biofilm formation. Our proteomics approach mirrored the phenotypic data as we identified differentially expressed cell wall proteins that correlate with the observed attenuated phenotypes. Our results show that Ddr48 is an important CWP influencing the architecture and proteomic constitution of the cell wall.

\section{Acknowledgments}

We would like to thank Dr. Brigitte Wex Dr. Georges Khazen, and Dr. Rony Khnayzer for their helpful advice and information.

\section{Conflict of Interest}

The authors have no financial conflicts of interest to declare.

\section{References}

1. Sorgo AG, Heilmann CJ, Dekker HL, Brul S. 2010. Mass spectrometric analysis of the secretome of Candida albicans. Yeast 201027: 661-672.

2. Southern P, Horbul J, Maher D, Davis DA. 2008. C. albicans colonization of human mucosal surfaces. PLoS One 3: e2067.

3. Viudes A, Pemán J, Cantón E, Úbeda P. 2002. Candidemia at a tertiary-care hospital: epidemiology, treatment, clinical outcome and risk factors for death. Eur. J. Clin. Microbiol. Infect. Dis. 21: 767-774.

4. Martins N, Ferreira IC, Barros L, Silva S. 2014. Candidiasis: predisposing factors, prevention, diagnosis and alternative treatment. Mycopathologia 177: 223-240.

5. Barada G, Basma R, Khalaf RA. 2008. Microsatellite DNA genotyping and identification of Candida albicans from Lebanese clinical isolates. Mycopathologia 165: 115-125.

6. Yazbek S, Barada G, Basma R, Mahfouz J. 2007. Significant discrepancy between real-time PCR identification and hospital identification of $C$. albicans from Lebanese patients. Med. Sci. Monit. 13: MT7-MT12.

7. Pfaller MA, Diekema DJ. 2007. Epidemiology of invasive candidiasis: a persistent public health problem. Clin. Microbiol. Rev. 20: 133-163.

8. Cheng S, Clancy CJ, Checkley MA, Handfield M. 2003. Identification of Candida albicans genes induced during thrush offers insight into pathogenesis. Mol. Microbiol. 48: 1275-1288.

9. Mayer FL, Wilson D, Hube B. 2013. Candida albicans pathogenicity mechanisms. Virulence 4: 119-28.

10. Jacobsen ID, Wilson D, Wächtler B, Brunke S. 2012. Candida albicans dimorphism as a therapeutic target. Expert. Rev. Anti. Infect. Ther. 10: 85-93.

11. Brown AJ, Odds FC, Gow NA. 2007. Infection-related gene expression in Candida albicans. Curr. Opin. Microbiol. 10: 307313.

12. Sudbery PE. 2011. Growth of Candida albicans hyphae. Nat. Rev. Microbiol. 9: 737-748.

13. Gow NA, Latge J, Munro CA. 2017. The fungal cell wall: structure, biosynthesis, and function. Microbiol. Spectr. 5(3). 
14. Masuoka J. 2004. Surface glycans of Candida albicans and other pathogenic fungi: physiological roles, clinical uses, and experimental challenges. Clin. Microbiol. Rev. 17: 281-310.

15. Tronchin G, Poulain D, Herbaut J, Biguet J. 1981. Localization of chitin in the cell wall of Candida albicans by means of wheat germ agglutinin. Fluorescence and ultrastructural studies. Eur. J. Cell. Biol. 26: 121-128.

16. Chaffin WL. 2008. Candida albicans cell wall proteins. Microbiol. Mol. Biol. Rev. 72: 495-544.

17. De Groot PW, Hellingwerf KJ, Klis FM. 2003. Genome-wide identification of fungal GPI proteins. Yeast 20: 781-796.

18. Erwig LP, Gow NA. 2016. Interactions of fungal pathogens with phagocytes. Nat. Rev. Microbiol. 14: 163-176.

19. Bitar I, Khalaf RA, Harastani H, Tokajian. 2014. Identification, typing, antifungal resistance profile, and biofilm formation of Candida albicans isolates from Lebanese hospital patients. Biomed. Res. Int. 2014: 931372.

20. Thomas DP, Viudes A, Monteagudo C, Lazzell AL. 2006. A proteomic-based approach for the identification of Candida albicans protein components present in a subunit vaccine that protects against disseminated candidiasis. Proteomics 6: 6033-6041.

21. Dib L, Hayek P, Sadek H, Beyrouthy B. 2008. The Candida albicans Ddr48 protein is essential for filamentation, stress response, and confers partial antifungal drug resistance. Med. Sci. Monit. 14: BR113-BR21.

22. Cleary IA, MacGregor NB, Saville SP, Thomas DP. 2012. Investigating the function of Ddr48p in Candida albicans. Eukaryot Cell 11: 718-724.

23. Awad A, El Khoury P, Wex B, Khalaf RA. 2018. Proteomic analysis of a Candida albicans pga1 null strain. EuPA Open Proteom. 18: 1-6.

24. Awad A, El Khoury P, Wex B, Khalaf RA. 2018. Tandem mass spectrometric cell wall proteome profiling of a Candida albicans hwp2 mutant strain. Curr. Mol. Pharmacol. 11: 211225.

25. El Khoury P, Awad A, Wex B, Khalaf RA. 2018. Proteomic analysis of a Candida albicans pir32 null strain reveals proteins involved in adhesion, filamentation and virulence. PLoS One 3: e0194403.

26. Zohbi R, Wex B, Khalaf RA. 2014. Comparative proteomic analysis of a Candida albicans DSE1 mutant under filamentous and non-filamentous conditions. Yeast 31: 441-448.

27. Pedreño Y, González-Párraga $P$, Martínez-Esparza $M$, Sentandreu R. 2007. Disruption of the Candida albicans ATC1 gene encoding a cell-linked acid trehalase decreases hypha formation and infectivity without affecting resistance to oxidative stress. Microbiology 153: 1372-1381.

28. Bahnan W, Koussa J, Younes S, Abi Rizk M. 2012. Deletion of the Candida albicans PIR32 results in increased virulence, stress response, and upregulation of cell wall chitin deposition. Mycopathologia 174: 107-119.
29. Plaine A, Walker L, Da Costa G, Mora-Montes HM. 2008. Functional analysis of Candida albicans GPI-anchored proteins: roles in cell wall integrity and caspofungin sensitivity. Fungal Genet. Biol. 45: 1404-1414.

30. Peeters E, Nelis HJ, Coenye T. 2008. Comparison of multiple methods for quantification of microbial biofilms grown in microtiter plates. J. Microbiol. Methods 72: 157-65.

31. Tsuchimori N, Sharkey LL, Fonzi WA, French SW. 2000. Reduced virulence of HWP1-deficient mutants of Candida albicans and their interactions with host cells. Infect. Immun. 68: 1997-2002.

32. Daher JY, Koussa J, Younes S, Khalaf RA. 2011. The Candida albicans Dse1 protein is essential and plays a role in cell wall rigidity, biofilm formation, and virulence. Interdiscip. Perspect. Infect. Dis. 2011: 504280.

33. Munro CA, Whitton RK, Hughes HB, Rella M. 2003. CHS8-a fourth chitin synthase gene of Candida albicans contributes to in vitro chitin synthase activity, but is dispensable for growth. Fungal. Genet. Biol. 40: 146-158.

34. Cabezón V, Llama-Palacios A, Nombela C, Monteoliva L. 2009. Analysis of Candida albicans plasma membrane proteome. Proteomics. 9: 4770-4786.

35. Barrett J, Brophy PM, Hamilton JV. 2005. Analysing proteomic data. Int. J. Parasitol. 35: 543-553.

36. Brasch J, Kreiselmaier I, Christophers E. 2003. Inhibition of dermatophytes by optical brighteners. Mycoses 46: 120-125.

37. Nodet P, Capellano A, Fevre M. 1990. Morphogenetic effects of Congo red on hyphal growth and cell wall development of the fungus Saprolegnia monoica. J. Gen. Microbiol. 136: 303310.

38. Ene I, Walker LA, Schiavone M, Lee KK. 2015. Cell wall remodeling enzymes modulate fungal cell wall elasticity and osmotic stress resistance. MBio 6: e00986-15.

39. Uppuluri P, Chaturvedi AK, Srinivasan A, Banerjee M. 2010. Dispersion as an important step in the Candida albicans biofilm developmental cycle. PLoS Pathog. 6: e1000828.

40. Frohner IE, Bourgeois C, Yatsyk K, Majer O. 2009. Candida albicans cell surface superoxide dismutases degrade hostderived reactive oxygen species to escape innate immune surveillance. Mol. Microbiol. 71: 240-252.

41. Rane HS, Bernardo SM, Hayek SR, Binder JL. 2014. The contribution of Candida albicans vacuolar ATPase subunit V $1 \mathrm{~B}$, encoded by $V M A 2$, to stress response, autophagy, and virulence is independent of environmental $\mathrm{pH}$. Eukaryot. Cell 13: 1207-1221.

42. Alberti-Sequi C, Morales AJ, Xing H, Kessler MM. 2004. Identification of potential cell-surface proteins in Candida albicans and investigation of the role of a putative cellsurface glycosidase in adhesion and virulence. Yeast 21: 285302.

43. Spreghini E, Davis DA, Subaran R, Kim M. 2003. Roles of Candida albicans Dfg5p and Dcw1p cell surface proteins in growth and hypha formation. Eukaryot. Cell. 2: 746-755. 
44. Klotz SA, Gaur NK, De Armond R, Sheppard D. 2007. Candida albicans Als proteins mediate aggregation with bacteria and yeasts. Med. Mycol. 45: 363-370.

45. Nobile CJ, Fox EP, Nett JE, Sorrells TR. 2012. A recently evolved transcriptional network controls biofilm development in Candida albicans. Cell 148: 126-138.

46. Nobile CJ, Andes DR, Nett JE, Smith FJ. 2006. Critical role of Bcr1-dependent adhesins in C. albicans biofilm formation in vitro and in vivo. PLoS Pathog. 2: e63.

47. Sentandreu M, Elorza MV, Sentandreu R, Fonzi WA. 1998. Cloning and characterization of PRA1, a gene encoding a novel pH-regulated antigen of Candida albicans. J. Bacteriol. 180: 282-289.

48. Peltroche-Llacsahuanga H, Goyard S, D'Enfert C, Prill SK. 2006. Protein O-mannosyltransferase isoforms regulate biofilm formation in Candida albicans. Antimicrob. Agents. Chemother. 50: 3488-3491.

49. Cornet M, Richard ML, Gaillardin C. 2009. The homologue of the Saccharomyces cerevisiae RIM9 gene is required for ambient $\mathrm{pH}$ signalling in Candida albicans. Res. Microbiol. 160: 219-223.

50. Bassilana M, Blyth J, Arkowitz RA. 2003. Cdc24, the GDPGTP exchange factor for $\mathrm{Cdc} 42$, is required for invasive hyphal growth of Candida albicans. Eukaryot. Cell 2: 9-18.

51. Almeida RS, Brunke S, Albrecht A, Thewes S. 2008. The hyphal-associated adhesin and invasin Als3 of Candida albicans mediates iron acquisition from host ferritin. PLoS Pathog. 4: e1000217. 